\title{
Role of Nurse in Postpartum Psychiatric Disorders
} \author{
TC $^{6}$ and Rajendra Badesgol ${ }^{7}$ \\ ${ }^{1}$ Department of Psychiatric Nursing DIMHANS Dharwad, India \\ ${ }^{2} 2$ nd Year MSc (Psychiatric Nursing) Student. DIMHANS Dharwad, India \\ ${ }^{3}$ Principal, Dhanush institute of Nursing Sciences Bagaklot \\ ${ }^{4}$ Staff Nurse, Indira Gandhi Children's Hospital, India \\ ${ }^{5}$ Assistant Professor, Department of Management Studies. Karnataka Arts College, India \\ ${ }^{6}$ Assistant Professor, RL Law College, India \\ ${ }^{7}$ Department of Police, India
}

Susheelkumar V Ronad ${ }^{1}$, Santosh S Ugargol ${ }^{2}$, Chetan S Patali ${ }^{3}$, Shridhar H Gondbal ${ }^{4}$, Kirankumar TC ${ }^{5}$, Pankaja

Submission: December 18, 2017; Published: January 04, 2018

*Corresponding author: Susheel Kumar V Ronad, Department of Psychiatric Nursing DIMHANS Dharwad, Karnataka, India, Email: usheelronad@gmail.com

\section{Introduction}

Women are at the greatest risk of developing a psychiatric disorder between the ages of 18 and 45 years Women in the puerperium are susceptible to the whole spectrum of psychiatric disorders. The bonds to the infant as well as the interaction with the baby are two aspects of the mother-infant relationship that can be disturbed by mothers with postpartum psychiatric disorders.

\section{Definition}

Postpartum psychiatric disorders pose a significant mental health problem in the community because of their prevalence and their impact of parent - infant and couple relationship [1]. The World Health Organization (WHO) designates the first 28 days after birth as the neonatal period. Although it has never been officially designated, the postpartum period is considered to start about an hour after the delivery of the placenta and is complete six weeks after birth. After six weeks, the mother's physical status will largely return to nonpregnant state in most instances. Any psychiatric symptoms appearing within six weeks period after delivery are called postpartum psychiatric disorders, if they do not fulfill the criteria of major psychiatric disorders. Postpartum psychiatric illness is divided into three main categories, postpartum depression, and postpartum psychosis

\section{Postpartum Blue}

Postpartum blues are transient, a self limiting condition with no known serious after effect. Most women recover from the blues within a day or two. It occurs at any time between the third and tenth postnatal day. It is considered as a normal reaction to child birth and affects and $70 \%$ to $80 \%$ of all postnatal mothers
[2]. These are common in Primigravida and in those who complain of premenstrual tension. Careful monitoring during this period is essential, since a small proportion of women with postpartum blues may develop postpartum, depression.

\section{Postpartum Depression}

Depression has been identified by the World Organization as a major cause of morbidity in the 21st century. The Global Burden of study states that major depression will become the second leading worldwide cause of en by 2020. Postpartum depression is a serious mood can cripple a woman's first month as a new mother. Postpartum depression is defined as, depression that occurs within weeks of postpartum (DSM-IV-TR). Postnatal the most frequent neurotic disorder during postnatal period and occurs at any point after the delivery with a peak of incidence within the first 4 months of postpartum. It occurs in 10\% - 15\% of women.

\section{Postnatal Psychosis}

The postpartum, psychosis is a most severe psychotic episode occurring during the postpartum period. Symptoms often begin as postpartum blues or postpartum depression. It is a rare event that occurs in approximately 1 to 2 per 1000 births and should be considered as a medical, psychiatric and obstetrical emergency [3]. Its presentation is often dramatic, with onset of symptoms as early as the first 48 to 72 hours after delivery. The majority of postpartum psychosis develops symptoms within the first two postpartum weeks.

a) What are postpartum psychiatric disorders?

b) List down the types of postpartum psychiatric disorders? 


\section{Summary}

You have completed Unit I of this structured teaching programme in this unit you have learn about the meaning of postpartum psychiatric disorders and its types.

\section{Ebiological Factors}

The postpartum period is generally regarded as a period of maturational crisis similar to the adolescence and menopause. The various stresses during the postpartum period include.
a) Endocrine change,
b) Changes of body images,
c) Activation of unconscious psychological conflicts.
d) Intra psychic recognition of becoming a mother.

On the light of these changes, etiological factors classifies into.
i. Biological
ii. Psychological
iii. Social and interpersonal factors

\section{Biological:}

Hereditary: Children of women treated for puerperal psychosis had a significantly higher prevalence of psychiatry disorders.

Endocrine Changes: The predominant explanation for the postpartum blue and depression is that levels of hormones such as? Progesterone, prolactine, and cortisol are either too high or too low in the puerperium, or that changes in the levels of these hormones occur too quickly so not quickly enough [4].

a) Hypothalamic - pituitary-gonadal axis: High progesterone, low estrogen and high prolactine levels are associated more often with affective disturbance especially blues. Progesterone and estrogen levels drop suddenly during the first 7-10 days postpartum, while prolactine level rise by ht 3rd day.

b) Cortisol: Abnormal cortisol levels are often associated with postpartum blues. Cortisol level increase during pregnancy, peak at birth and decline suddenly after childbirth.

\section{Biochemical factor}

a) Cyclic adenosine monophosphate (cAMP): low cAMP level results in postpartum repression.

b) Amines: Low levels of serotonin and tryptophan and high level of nor-metanephirne have found in postpartum blues.

c) Endorphins: Decreased level of endorphins is associated with mood changes. d) Serum calcium: elevated serum calcium results in postpartum psychosis

e) Sleep pattern changes

f) Changes in the sleep patterns may account for the patient's postpartum vulnerability. Just prior to deliver, state 4 NREM sleep is reduced and does return to normal until the second week postpartum.

\section{Psychological Factors}

Psychodynamic Explanations: Gender bias include preference to male child, presence of conflicting feeling within the mother with regard to her mothering experience, her new baby, her husband and herself.

Personality factory: Increased number of infantile and immature traits in the premorbid personalities.

\section{Social and Interpersonal Factors:}
a) Violence against women
b) Economic deprivation
c) Husband's supportive role and practices affect the psychological need of mother.
d) Poor social support.
i. List down the stressors during postpartum period?
ii. What are the biological factors of postpartum psychiatric disorders?
iii. What are the psychological factors of postpartum psychiatric disorders?

\section{Summary}

You have completed unit IV of this module in this unit you have learn about the etiologic factors of postpartum disorders.

\section{Classification}

\section{ICD 10 Classification}

a) F53 Mental and behavioral disorders associated with the puerperium, not elsewhere classified. This classification should be used only for mental disorders associated with the puerperium (commencing within 6 weeks of delivery) that do not meet the criteria for disorders classified elsewhere. It will usually be possible to classify mental disorders associated with the puerperium by using two other codes: the first is from elsewhere in Chapter V (F) and indicates the specific type of mental disorder (usually affective (F30-F39) and the second of ICD-10 [5].

b) F53:0 Mild mental and behavioral disorders associated with the puerperium, not elsewhere classified.

a. Includes: Postnatal depression NOS, postpartum, depression NOS. 
c) F53.1 seven mental and behavioral disorders associated with the puerperium, not elsewhere classified.

a. Includes: Puerperal psychosis NOS

d) F53.8 other mental and behavioral disorders associated with the puerperium, not elsewhere classified.

e) F 53.9 Puerperal mental disorders, unspecified.

\section{DSM-IV Classification}

According to DSM-IV, postpartum psychiatric illnesses may be indicated with a postpartum onset specifies can be applied to the current or most recent major depressive, manic, or mixed episode in major depressive disorders, bipolar I bipolar II, or brief psychotic disorders.

Classification by Inwood: Inwood has classified postpartum psychiatric disorders into 3 types.

a) Type 1: postpartum psychosis (puerperal psychiatric disorders into 3 types.

b) Type 2: Adjustment reaction with depressed mood (also called as postpartum, blues/maternal blues or postpartum perplexity syndrome)

c) Type 3: Postpartum major mood disorder (also called as postpartum neurosis or neurotic reaction).

\section{Common Classification:}
a) Postpartum blues
b) Postpartum depression
c) Postpartum psychosis

\section{Section - II Review questions:}

a) What are the common classifications of postpartum psychiatric disorders?

b) What are the classifications of postpartum psychiatric disorders by Inwood?

Summary: You have completed unit III of this module, in this unit you have learn about the classifications of postpartum psychiatric disorders.

\section{Section - I Clinical features}

\section{Post partum blues}
a) Sadness
b) Mood liability
c) fearfulness
d) Anxiety
e) Irritability
f) Inability to sleep
g) feeling of loneliness
h) Confusion
i) Fatigue

\section{Post partum depression}
a) Depressed or sad mood
b) Fearfulness
c) Loss of interest in daily activities
d) feeling of guilt
e) feeling of worthlessness or incompetence
f) Fatigue
g) sleep disturbance
h) Change in appetite
i) Poor concentration
j) Suicidal thoughts
k) Lack of enjoyment in the maternal role

\section{Post partum psychosis}

a) Symptoms often begin with postpartum blues or postpartum depression

b) Delusions

c) Hallucinations

d) Confusion

e) Delirium

f) Panic

g) Suicide or infanticide

h) Restlessness

i) Irritability

j) Rapidly shifting depressed or elated mood

k) Disorganized behavior

I. What are clinical features of postpartum blues?

II. What are clinical features of postpartum depression?

\section{Summary}

You have completed unit IV of this module, in this unit you have learn about the clinical features of postpartum psychiatric disorders.

\section{Prevention and treatment}

Initial evaluation should include a thorough history (antenatal, intranatal), physical examination and routine laboratory tests. The clinical evaluation should include CBC, complete blood chemistry, thyroid function and antithyroid antibody tests, and calcium, vitamin B12 and folate levels. 
a) Postpartum blues: Patients with postpartum blues have mild and transient symptoms, which remit spontaneously, it therefore requires on specific and transient or a psychiatric consultation. Support and reassurance is the mainstay of treatment.

b) Postpartum depression: 1 e early initiation of treatment results in and better prognosis women with postpartum depression are treated on the same guidelines nonpuerperal depressed patients. The management can be grouped as;

\section{Non - pharmacological:}

a) Debriefing: It is brief version of client-centered therapy for postpartum depression using health visitors over a period of 8 weeks. Debriefing is being introduced for women after childbirth with the aim of improving psychological recovery. This gives women an opportunity to discuss their experiences of delivery with an empathetic listener.

b) Cognitive behave Therapy: It is a psychotherapeutic approach based on the idea that behavior is secondary to thinking. Short-term cognitive-behavioral therapy (CBT) was as effective as treatment with Fluoxetine in women with postpartum depression.

c) Interpersonal therapy: It is effective for the treatment of women with mild to moderate postpartum depression and improving the quality of their interpersonal relationship.

d) Good supervision support

e) Counseling

\section{Pharmacological:}

a) Antidepressant medications: Fluoxetine, setraline, fluvoxamine amitriptyline and venlafaxine.

b) Mood stabilizers : lithium, carbamazepine and valporic acid

c) Benzodiazepines anti anxiety medications : clonazepam. Lorazepam

d) Tricyclic antidepressants: impipramine, clomipramine

e) Electro-convulsive therapy : It is the artificial induction of grandmal seizure through the application of electric current to the brain

f) Hormonal Therapy

g) Progesterone alone

h) Combination of estrogen and antidepressant: $17 \AA$ estradiol 200mg daily and dydrogesterone $10 \mathrm{mg}$

\section{Postpartum psychosis:}

a) Admission to the hospitals always required due to potential danger to the baby and difficulty in dealing with mothers behavior in home. b) Electroconvulsive there Electroconvulsive therapy (ECT) is well tolerated and rapidly effective for severe postpartum depression and psychosis.

c) Antipsychotics: Haloperidol (Haldol) $5 \mathrm{mg} /$ day

d) Supportive Psychotherapy

\section{Nurses responsibilities:}

ANM students play a crucial role in identifying and interventions for postpartum psychiatric disorder and recognize the impact on women and their families are minimized through acknowledgement, support and education the postpartum psychiatric disorders clients to improve the awareness of postpartum psychiatric disorders and strengthen the relationship between parents and infants.

a) Ensure effective communication and understand detail of the client specially including the both antenatal and postnatal.

b) Identify the risk factors associated with postpartum psychiatric disorders including prenatal depression child care stress life stress lack of social support prenatal anxiety maternity blues marital dissatisfaction history of previous depression difficult infant temperament low self-esteem low socioecomic status, unplanned/unwanted pregnancy, single marital status preterm birth and multiple birth.

c) Nurse should be recognize the symptoms for the spectrum of postpartum psychiatric disorders ranging from baby blues" to life - threatening postpartum psychoses and being knowledgeable about the range of treatment options available for the variety of postpartum psychiatric disorders.

d) Nurse should assess the women thoughts and ensure the safety of mother and her child and delusions, compounded with feelings of irritability and difficulty in controlling emotions psychiatric disorders.

e) Nurse must be alert for signs of dysfunction and be prepared to help promote attachment between mother and baby, referral of the mother and family for support services and counseling and assisting the family prioritizing and performing necessary family functions

f) Assist the mother in breast feeding techniques.

g) Maintain a therapeutic relationship and a community

h) Maintain a detail and comprehensive understanding of the depth, types and breadth of the postpartum psychiatric disorders

i) An open discussion should be concerns with using pharmacotherapy and the benefits and risks of treatment when breastfeeding.

j) Nurse should educate the mothers about available services if symptoms services if symptoms develop and of the serious consequences of untreated illness. 
k) Ensure about adequate antenatal check up and cares are provided.

l) Nurse should have adequate I knowledge about Edinburgh postnatal Depression Scale and the ability to practicing it.

m) Nurse should be maintaining a collaborative approach among all health professionals is essential in order to detect and most effectively manage postpartum psychiatric disorders receive a greater place in nursing curriculums, as well as for ongoing educational programs for all health professional.

n) Nurse should obtaining knowledge about the distinctions among postpartum psychiatric disorders to differentiate postpartum depression from other mental health illnesses occurring after birth.

o) Proper education given regarding postpartum period and follow up care.

Nurses serve a vital role in maximizing the health and health care experiences of pregnant women and new mothers and encourages federal and private health insurance plans to provide appropriate treatment options to women suffering with postpartum psychiatric disorders [6].

A. What are preventive measures of postpartum depression?

B. What are preventive measures of postpartum psychosis?

\section{Summary}

You have completed unit $\mathrm{V}$ of this module, in this unit you have learn about the preventive and treatment measures of postpartum psychiatric disorders.

\section{Answers to Review Questions}

\section{Definition}

A. What are postpartum psychiatric is disorders?

Any psychiatric symptoms appearing within six weeks period after delivery are called postpartum psychiatric disorders, if they do not fulfill the criteria of major psychiatric disorders.

B. List down the types of postpartum psychiatric disorders?
a) Post partum blues
b) Post partum depression
c) Post partum psychosis

\section{Etiology}

A. List down the stressors during postpartum period?

a) Endocrine Changes, b) Changes of body image

c) Activation of unconscious psychological conflicts

d) Intra psychic recognition of becoming a mother.

B. What are the biological factors postpartum psychiatric disorders?

a) Hereditary

b) Endocrine changes

c) Biochemical factors

d) Sleep pattern changes

C. What are the social and interpersonal factors of postpartum psychiatric disorders?

a) Violence against women

b) Economic deprivation

c) Obstetrical practices affect the psychological needs of mother

d) Poor social support

\section{Classification}

A. What are the common classifications of postpartum psychiatric disorders?

a) Postpartum blues.

b) Postpartum depression

c) Postpartum psychosis

B. What are the classification postpartum psychiatric disorders by Inwood?

a) Type 1: Postpartum psychosis (puerperal psychosis or brief reactive psychosis)

b) Type 2: Adjustment reaction with depressed mood (also called as postpartum blues maternal blues of postpartum perplexity syndrome).

c) Type 3: Postpartum major mood disorder (also called as postpartum neurosis neurotic reaction).

\section{Classification}

A. What are clinical features of postpartum blues?

a) Sadness

b) Mood liability

c) Tearfulness

d) Anxiety

e) Inability

f) Inability to sleep

g) Feeling of loneliness 

h) Confusion
i) Fatigue
B. What are clinical features of postpartum depression?
a) Depressed or sad mood
b) Tearfulness
c) Loss of interest in daily activities
d) Feeling of guilt
e) Feeling of worthlessness or incompetence
f) Fatigue
g) Sleep disturbance
h) Change in appetite
i) Poor Concentration
j) Suicidal thoughts
k) Lack of enjoyment in the maternal role

\section{Prevention and Treatment}

A. What are preventive measures of postpartum depression?
a) Non-pharmacological:
i. Debriefing
ii. Cognitive behavioral therapy
iii. Interpersonal therapy
iv. Good supervision and support
v. Counseling

\section{b) Pharmacological:}

i. Antidepressant medications

ii. Benzodiazepines anti anxiety medications

iii. Tricyclic antidepressants

iv. Electro - convulsive therapy

c) Hormonal Therapy:

i. Progesterone

ii. Combination of estrogen and an antidepressant.

B. What are preventive measures of postpartum psychosis?

a) Admission to the hospitals always required due to potential danger to the baby and difficulty in dealing with mothers behavior in home.

b) Electroconvulsive the

c) Antipsychotics.

d) Supportive psycho therapy

\section{References}

1. Sampoornam W (2011) Postpartum mothers knowledge of mental problems. Nightingale nursing times. (7): 6-10.

2. Shubhangini Parker, Smita A pandit, Shah LP Text book of post graduate psychiatry. 1(1): 369-375.

3. Niraj A (2006) A short text book of Jaypee $5^{\text {th }}$ edn $152-153$.

4. Sreevani R A guide to mental health and psychiatric nursing, Jaypee $2^{\text {nd }}$ edn, 248-250.

5. Postpartum Psychiatric Disorders.

6. Bobak, lenson, Lowder Milk Maternity nursing $4^{\text {th }}$ edn. 664-649.

\section{Your next submission with Juniper Publishers} will reach you the below assets

- Quality Editorial service

- Swift Peer Review

- Reprints availability

- E-prints Service

- Manuscript Podcast for convenient understanding

- Global attainment for your research

- Manuscript accessibility in different formats ( Pdf, E-pub, Full Text, Audio)

- Unceasing customer service

Track the below URL for one-step submission https://juniperpublishers.com/online-submission.php 